\title{
Diocotron instability in pulsar electrospheres
}

\section{Linear analysis}

\author{
J. Pétri ${ }^{1}$, J. Heyvaerts ${ }^{1}$, and S. Bonazzola ${ }^{2}$ \\ 1 Observatoire de Strasbourg, 11 rue de l'Université, 67000 Strasbourg, France \\ 2 DARC, Observatoire de Meudon, Place Jules Janssen, 92195 Meudon, France
}

Received 22 October 2001 / Accepted 8 March 2002

\begin{abstract}
In a previous work, we have shown that the electrospheric plasma of a pulsar's magnetosphere with vacuum gaps is subject to differential rotation in the equatorial belt. Here we examine the stability of a simplified model of this belt. The model consists of a charged thin plasma disc in differential rotation, embedded in a dipolar magnetic field anchored in the neutron star. In the linearized electrostatic perturbation approximation, this disc appears to be unstable to the so-called diocotron instability. We present several eigenspectra and eigenfunctions for different disc models, which differ by the total charge of the disc-star system. Velocity and electric field perturbations are also shown in the disc plane for a few fast-growing modes. Increasing the total charge has a stabilizing effect, in the sense that the growth rate of the fastest-growing eigenvalue decreases with total charge and eventually vanishes. When the system is unstable, the growth rates are of the order of the azimuthal rotation rate. This means that the diocotron instability, which develops in a few pulsar's periods, should be very efficient in causing particles to migrate across magnetic surfaces.
\end{abstract}

Key words. instabilities - magnetic fields - MHD - methods: numerical - stars: pulsars: general - plasmas

\section{Introduction}

An isolated neutron star rotating about its dipole axis may be surrounded by a charged magnetosphere in electrostatic equilibrium with large vacuum gaps (Krause-Polstorff \& Michel 1985; Smith et al. 2001; Petri et al. 2002), which we refer to as an electrosphere. The electrosphere consists of polar domes, corotating with the star and of a charged differentially rotating equatorial disc. Electrospheres in equilibrium would not show any form of electromagnetic activity. They correspond to dead pulsars. However, their stability should be questioned, because the existence of a differentially rotating disc could give rise to a shearing instability. If an instability grows, its non-linear development would give rise to charged particle diffusion across magnetic surfaces. Some form of electric current circulation, otherwise forbidden, would then be made possible and could eventually give rise to some electromagnetic activity.

Similar situations are important in other contexts. Bohm diffusion, a fundamental process in laboratory plasma confinement, results from particle diffusion across field lines induced by unstable drift waves (Horton 1999). In astrophysical situations associated with accretion discs,

Send offprint requests to: J. Pétri,

e-mail: petri@newbr.u-strasbg.fr an instability similarly allows viscous accretion of the plasma onto the mass-receiving star. This instability is thought to be the magnetorotational instability (Balbus \& Hawley 1991), which develops in weak fields with a maximal growth rate of the same order of magnitude as the angular rotation velocity. At the boundary between the magnetosphere of a magnetized star and a surrounding accretion disc a global interchange instability of an electromagnetic nature may similarly allow plasma transport and accretion (Stehle \& Spruit 2001).

It is therefore very important to examine whether neutron star electrospheres with vacuum gaps are subject to large-scale instabilities of their differentially rotating equatorial belt, since this would allow particle motions across magnetic surfaces. Similar ideas are considered by Spitkovsky \& Arons (2002). Such large-scale instabilities are not to be confused with the widely studied plasma micro-instabilities in which the coherent radio radiation emitted by particle beams streaming off the polar caps of active pulsars is thought to originate (Arons \& Smith 1979; Asséo et al. 1983; Weatherall 1997). The large-scale instabilities of the electrospheric equatorial disc to be considered below share similarities both with the Kelvin Helmholtz instability, because differential rotation is involved, and with drift wave instabilities, because electric drift controls plasma 
motions and the plasma density is inhomogeneous. The physical regime and the geometry are nevertheless quite different in pulsars' electrospheres as compared to Kelvin Helmholtz flows. Inertial wave instabilities in rotating plasma columns have been considered in the context of the polar cap regions of active pulsars by Mofiz (1992). In this situation, which is also different to the one considered here, differential rotation is neglected and the plasma is a relativistically hot lepton plasma with the geometry of a cylindrical column.

Unlike dense non-relativistic accretion discs and laboratory confinement machines, but similarly to some electronic devices (Davidson 1990), the pulsar electrosphere is a non-neutral medium. The expected instabilities should then be studied in the relevant regime of a charged plasma, the motion of which is controlled by electric drift. The geometry of the system to be studied should be relevant to the actual structure of the electrosphere. In this paper we discuss the linear stability of a flat charged disc embedded in a dipolar magnetic field. We will study the nonlinear development of the instability that we have discovered in a forthcoming paper.

\section{The thin disc approximation}

For simplicity, we reduce the structure of the pulsar's electrosphere to its simplest form: a differentially rotating thin equatorial disc embedded in the dipolar neutron star's magnetic field. This thin disc is self-consistently constructed. To avoid non-essential complications due to the electric reaction of the domes that extend over the polar regions of the neutron star, these are simply removed from the model, and replaced by surface charge density present on the star's surface.

We consider motions in the equatorial plane only. The plasma flow is then purely two-dimensional but the structure of the electric field remains three dimensional.

The disc is treated as a cold, non-relativistic, chargeseparated plasma consisting of particles of mass $m_{q}$ and charge $q$. The diocotron regime occurs when the plasma frequency, given by $\omega_{\mathrm{p}}^{2}=n_{q} q^{2} / m_{q} \varepsilon_{0}$, is negligible compared to the cyclotron frequency $\omega_{\mathrm{c}}=q B / m_{q}$, that is, when

$\frac{\omega_{\mathrm{p}}^{2}}{\omega_{\mathrm{c}}^{2}} \ll 1$.

Although our model represents the equatorial belt as an infinitely thin plasma disc, we nevertheless assume that the actual disc thickness is large enough for this inequality to be satisfied. The diocotron frequency is defined as

$\omega_{\mathrm{D}}=\frac{\omega_{\mathrm{p}}^{2}}{2 \omega_{\mathrm{c}}}$.

It corresponds to the period of a circular motion at the slow electric drift velocity $\boldsymbol{E} \times \boldsymbol{B} / B^{2}$ (Davidson 1990). The low density approximation can be restated in the form:

$n_{q} m_{q} c^{2} \ll \frac{B^{2}}{\mu_{0}}$.
It implies that the rest mass energy density of the plasma should be less than the magnetic energy density. In the sub-relativistic approximation, plasma currents have no significant effect on the external magnetic field. The latter can therefore be considered constant. Thus the perturbation is of an electrostatic nature. It is straightforward to show that Eq. (3) is always fulfilled for plasma confined well within the light cylinder. Indeed the dipolar magnetic field decreases with distance as $1 / R^{3}$ and, for a density equal to the Goldreich-Julian charge density $n_{q}=\varepsilon_{0} \Omega_{*} B / q$, Eq. (3) implies that

$R \ll\left(\frac{q B_{*}}{m_{q} \Omega_{*}}\right)^{1 / 3} R_{*}$

Evaluating the term on the right of this equation for electrons or positrons in the field of a pulsar of a period of the order of a second, (with typical parameters $B_{*}=10^{8} \mathrm{~T}$ and $\Omega_{*}=2 \pi \mathrm{rad} / \mathrm{s}$ ), we find $\left(\frac{q B_{*}}{m_{q} \Omega_{*}}\right)^{1 / 3} \approx 1.4 \times 10^{6}$. For comparison, the radius of the light cylinder is $R_{\mathrm{L}} \approx$ $5000 R_{*}$. For millisecond pulsars $R_{\mathrm{L}} \approx 5 R_{*}$. Then in this case the electrostatic approximation is not valid and the magnetic field perturbation caused by the electrospheric currents should be taken into account.

\section{Linearized equations for small perturbations}

\subsection{Equilibrium state}

We analyze the diocotron instability in the frame of linear perturbation theory. A small perturbation is applied to the system, starting from an equilibrium situation. Linearizing, we obtain a system of equations that we reduce to an unique eigenvalue equation for the perturbation. The eigenspectrum then reveals the presence of an instability, if any.

The equilibrium is an electrodynamically selfconsistent state. It satisfies the continuity equation, Poisson's equation and the equation of the motion, written in the electric drift approximation, which we write as ${ }^{1}$

$$
\begin{cases}\nabla \cdot\left(\sigma_{0} \boldsymbol{v}_{0}\right) & =0 \\ \varepsilon_{0} \Delta \Phi_{0} & =-\sigma_{0} \delta(z) \\ \boldsymbol{v}_{0} & =\frac{\boldsymbol{E}_{0} \times \boldsymbol{B}_{0}}{B_{0}^{2}} \\ \boldsymbol{E}_{0} & =-\boldsymbol{\nabla} \Phi_{0}\end{cases}
$$

The potential $\Phi_{0}$ is the sum of the potential of the star, denoted by $\Phi_{*}$ (which is, and remains, fully axisymmetric since no perturbation reaches the pulsar's interior or its surface), and of the potential of the disc, denoted by $\Phi_{\mathrm{D}}$. Since in our approach the magnetic field created by the electrospheric current is neglected, the magnetic field remains constant and dipolar, and is given by:

$\boldsymbol{B}=\boldsymbol{B}_{0}=B_{0} \boldsymbol{e}_{z}=-\frac{1}{2} \frac{B_{*} R_{*}^{3}}{r^{3}} \boldsymbol{e}_{z}$.

\footnotetext{
1 In the following, we use the subscript 0 for the unperturbed quantities and the subscript 1 for the first order perturbations.
} 


\subsection{The perturbed state}

The general time-varying behavior of the non-neutral plasma in response to an electrostatic perturbation is described by the equations

$$
\begin{cases}\nabla \cdot(\sigma \boldsymbol{v})+\frac{\partial \sigma}{\partial t} & =0 \\ \varepsilon_{0} \Delta \Phi & =-\sigma \delta(z) \\ \boldsymbol{v} & =\frac{\boldsymbol{E} \times \boldsymbol{B}}{B^{2}} \\ \boldsymbol{E} & =-\stackrel{\nabla}{\nabla} .\end{cases}
$$

This system is linearized by expanding all scalar and vectorial component quantities $\psi$ about the equilibrium state (5) to first order in the perturbations, like $\psi \approx$ $\psi_{0}+\psi_{1}$, with $\psi_{1} \ll \psi_{0}$. Noting that the electrospheric current $\boldsymbol{\nabla} \times \boldsymbol{B}_{0}=0$, we obtain, after some algebra, the potential/density pair relations:

$$
\left\{\begin{aligned}
\boldsymbol{v}_{0} \cdot & \boldsymbol{\nabla} \sigma_{1}-\nabla \sigma_{0} \cdot\left(\boldsymbol{\nabla} \Phi_{1} \times \frac{\boldsymbol{B}_{0}}{B_{0}^{2}}\right) \\
& +\sigma_{0} \nabla \Phi_{1} \cdot\left(\boldsymbol{\nabla} \frac{1}{B_{0}^{2}} \times \boldsymbol{B}_{0}\right)+\frac{\partial \sigma_{1}}{\partial t}=0 \\
\sigma_{1}= & -\varepsilon_{0} \Delta \Phi_{1} .
\end{aligned}\right.
$$

In our particular geometry, the magnetic field is parallel to the common direction of the rotation axis and magnetic moment, and the equilibrium plasma motion reduces to an azimuthal rotation with velocity $\boldsymbol{v}_{0}=$ $r \Omega_{0}(r) \boldsymbol{e}_{\varphi}$. Neglecting the induced and displacement current $\left(\varepsilon_{0} \frac{\partial \boldsymbol{E}}{\partial t}\right)$, the system (8) can be expressed in polar coordinates $(r, \varphi)$, as:

$$
\left\{\begin{array}{l}
\Omega_{0} \frac{\partial \sigma_{1}}{\partial \varphi}-\frac{B_{0}}{r} \frac{\mathrm{d}}{\mathrm{d} r}\left(\frac{\sigma_{0}}{B_{0}^{2}}\right) \frac{\partial \phi_{1}}{\partial \varphi}+\frac{\partial \sigma_{1}}{\partial t}=0 \\
\Delta \Phi_{1}=-\frac{\sigma_{1}}{\varepsilon_{0}} \delta(z)
\end{array}\right.
$$

These equations describe the evolution of any infinitesimal density perturbation. We solve them by Fourier analysis as described in the next section.

\section{The eigenvalue problem}

\subsection{The differential equation}

To any physical quantity $\psi(r, \varphi, t)$ in the disc let us associate a complex function $\chi(r, \varphi, t)$ such that $\psi=\operatorname{Re}(\chi)$, which we further expand as:

$$
\chi(r, \varphi, t)=\tilde{\chi}(r) \mathrm{e}^{i(m \varphi-\omega t)} .
$$

The frequency $\omega$ is a complex number and $m$ is an integer. Denoting the real part with a subscript $r$ and the imaginary part with a subscript $i$, the real physical quantity $\psi$ is given in terms of the complex quantity $\tilde{\chi}$ by:

$$
\begin{aligned}
\psi(r, \varphi, t)= & {\left[\tilde{\chi}_{r}(r) \cos \left(m \varphi-\omega_{r} t\right)\right.} \\
& \left.-\tilde{\chi}_{i}(r) \sin \left(m \varphi-\omega_{r} t\right)\right] \mathrm{e}^{\omega_{i} t} .
\end{aligned}
$$

The amplitude of the perturbation grows or decays exponentially with time, depending on the sign of the growth rate $\gamma=\omega_{i}=\operatorname{Im}(\omega)$. An instability occurs when the growth rate is positive. The perturbation described by Eq. (10) rotates at the "pattern" angular frequency $\Omega_{\mathrm{p}}=$ $\frac{\operatorname{Re}(\omega)}{m}$. The corotation radius, $r_{\mathrm{c}}$, is the radius at which this frequency equals the local rotation rate of the plasma in equilibrium: $\Omega_{0}\left(r_{\mathrm{c}}\right)=\Omega_{\mathrm{p}}$. Note that the radius $r_{c}$ is not always uniquely determined. Using Eqs. (10) and (11) the system (9) is transformed into

$$
\left\{\begin{array}{l}
\frac{1}{r} \frac{\partial}{\partial r}\left(r \frac{\partial \tilde{\Phi}_{1 m}}{\partial r}\right)+\frac{\partial^{2} \tilde{\Phi}_{1 m}}{\partial z^{2}}-\frac{m^{2}}{r^{2}} \tilde{\Phi}_{1 m}=-\frac{\tilde{\sigma}_{1 m}}{\varepsilon_{0}} \delta(z) \\
\left(\omega-m \Omega_{0}\right) \tilde{\sigma}_{1 m}+\frac{m}{r} B_{0}\left(\frac{\mathrm{d}}{\mathrm{d} r} \frac{\sigma_{0}}{B_{0}^{2}}\right) \tilde{\Phi}_{1 m}=0
\end{array}\right.
$$

Replacing $\tilde{\sigma}_{1}$ from the second equation into the first one, we obtain the eigenvalue equation for a given mode $m$ in the form of an equation for the potential perturbation only:

$$
\begin{gathered}
\frac{1}{r} \frac{\partial}{\partial r}\left(r \frac{\partial \tilde{\Phi}_{1 m}}{\partial r}\right)+\frac{\partial^{2} \tilde{\Phi}_{1 m}}{\partial z^{2}}-\frac{m^{2}}{r^{2}} \tilde{\Phi}_{1 m}= \\
\frac{m}{\omega-m \Omega_{0}} \frac{B_{0}}{\varepsilon_{0}} \frac{1}{r}\left(\frac{\mathrm{d}}{\mathrm{d} r} \frac{\sigma_{0}}{B_{0}^{2}}\right) \delta(z) \tilde{\Phi}_{1 m} .
\end{gathered}
$$

The boundary conditions are that $\tilde{\Phi}_{1}$ vanishes at infinity and on the star's surface. This equation apparently depends on three given functions: the rotation speed $\Omega_{0}(r)$, the magnetic field $B_{0}(r)$ and the charge density $\sigma_{0}(r)$ at equilibrium. In fact, only two of them are independent because they are self-consistently related by Eq. (5).

\subsection{The Fredholm integral equation}

Because of the presence of a Dirac distribution on the right hand side, Eq. (13) cannot be numerically solved by classical methods, such as, for example, the shooting method. This method is appropriate only for differential equations without singularities. To overcome this difficulty, we get rid of the Dirac distribution by a direct integration of the Poisson equation, taking into account the particular boundary conditions that apply to our problem. This is done by using the relevant Green function. Details of this calculation are described in Appendix A. Selecting a particular value of the azimuthal mode number $m$, the perturbed potential is thus expressed as

$$
\begin{aligned}
\tilde{\Phi}_{1 m}(r)= & \frac{1}{2 \varepsilon_{0}} \int_{R_{*}}^{\infty} \int_{0}^{\infty}\left[J_{m}(k r) J_{m}\left(k r^{\prime}\right)\right. \\
& \left.-\frac{R_{*}}{r^{\prime}} J_{m}(k r) J_{m}\left(k \frac{R_{*}^{2}}{r^{\prime}}\right)\right] \mathrm{d} k \tilde{\sigma}_{1 m}\left(r^{\prime}\right) r^{\prime} \mathrm{d} r^{\prime}
\end{aligned}
$$

where $k$ is the wave number in the vertical direction.

For $m \neq 0$ there is no contribution from the star, because the stellar potential is independent of $\varphi$ and is not subject to any perturbation. The expression in square brackets represents the potential at $(r, z=0)$ from a ring with unit charge at $\left(r^{\prime}, z=0\right)$. This potential vanishes at $R=R_{*}$ and at infinity, as required. It has a logarithmic singularity when the point $r$ and the source point $r^{\prime}$ coincide. Inserting Eq. (14) in the continuity Eq. (12), we 
$A=\left[\begin{array}{cccc}\left(m \Omega_{1}+R_{1}-\sum_{j \neq 1} w_{j} K_{1 j}\right) & \cdots & w_{j} K_{1 j} & w_{n} K_{1 n} \\ \vdots & \cdots & \ldots & \vdots \\ w_{1} K_{i 1} & \left(m \Omega_{i}+R_{i}-\sum_{j \neq i} w_{j} K_{i j}\right) & w_{j} K_{i j} & w_{n} K_{i n} \\ \vdots & \ldots & \ldots & \vdots \\ w_{1} K_{n 1} & \cdots & w_{n-1} K_{n-1, n} & \left(m \Omega_{n}+R_{n}-\sum_{j \neq n} w_{j} K_{n j}\right)\end{array}\right]$.

obtain the eigenvalue equation satisfied by the perturbed density $\tilde{\sigma}_{1 m}$ :

$\omega \tilde{\sigma}_{1 m}(r)=m \Omega_{0}(r) \tilde{\sigma}_{1 m}(r)+\int_{r_{1}}^{r_{2}} K_{m}\left(r \mid r^{\prime}\right) \tilde{\sigma}_{1 m}\left(r^{\prime}\right) \mathrm{d} r^{\prime}$.

Equation (15) is a homogeneous Fredholm integral equation of the third kind. The kernel is given by

$$
\begin{aligned}
K_{m}\left(r \mid r^{\prime}\right)= & -\frac{m}{2} \frac{r^{\prime}}{r} \frac{B_{0}}{\varepsilon_{0}} \frac{\mathrm{d}}{\mathrm{d} r}\left(\frac{\sigma_{0}}{B_{0}^{2}}\right) \int_{0}^{\infty}\left[J_{m}(k r) J_{m}\left(k r^{\prime}\right)\right. \\
& \left.-\frac{R_{*}}{r^{\prime}} J_{m}(k r) J_{m}\left(k \frac{R_{*}^{2}}{r^{\prime}}\right)\right] \mathrm{d} k
\end{aligned}
$$

It has a logarithmic singularity on the diagonal $r=r^{\prime}$, and can be expressed in closed analytical form in terms of the hypergeometric ${ }^{2}$ functions ${ }_{2} F_{1}$ :

$$
\begin{aligned}
K_{m}\left(r \mid r^{\prime}\right)= & -\frac{m}{2} \frac{r^{\prime}}{r} \frac{B_{0}}{\varepsilon_{0}} \frac{\mathrm{d}}{\mathrm{d} r}\left(\frac{\sigma_{0}}{B_{0}^{2}}\right) \frac{\Gamma\left(m+\frac{1}{2}\right)}{\Gamma(m+1) \Gamma\left(\frac{1}{2}\right)} \\
& {\left[\frac{r_{<}^{m}}{r_{>}^{m+1}}{ }_{2} F_{1}\left(m+\frac{1}{2}, \frac{1}{2} ; m+1 ; \frac{r_{<}^{2}}{r_{>}^{2}}\right)^{2}-\right.} \\
& \left.\frac{R_{*}^{2 m+1}}{\left(r r^{\prime}\right)^{m+1}}{ }_{2} F_{1}\left(m+\frac{1}{2}, \frac{1}{2} ; m+1 ; \frac{R_{*}^{2}}{\left(r r^{\prime}\right)^{2}}\right)\right]
\end{aligned}
$$

We have introduced the notation $r_{<}=\min \left(r, r^{\prime}\right)$ and $r_{>}=\max \left(r, r^{\prime}\right)$. The kernel can be written as $K_{m}\left(r \mid r^{\prime}\right)=$ $f(r) G_{m}\left(r \mid r^{\prime}\right) r^{\prime}$. It is clearly not symmetrizable when $f(r)$ is not a constant, as is obviously the case here. Thus no analytic solution can be found. A numerical solution is necessary.

\section{Numerical method}

The numerical determination of the eigenvalues and eigenvectors of a homogeneous Fredholm integral equation of the second kind

$f(x)=\lambda \int_{a}^{b} K(x, t) f(t) \mathrm{d} t$

with a regular kernel $K$ on a finite interval $[a, b]$ is straightforward. The Nystrom method involves the choice of some

\footnotetext{
${ }^{2}$ See the properties of Bessel functions as given in the mathematical table of Abramowitz \& Stegun (1965).
}

quadrature formula with associated weights $w_{i}$ and abscissae $t_{i}$ to estimate the integral at the right of Eq. (15) by

$\int_{a}^{b} K(x, t) f(t) \mathrm{d} t \approx \sum_{i=1}^{N} w_{i} K\left(x, t_{i}\right) f\left(t_{i}\right)$

Let $f_{i}$ be the value of $f$ at the quadrature points $t_{i}$ and $K_{i j}$ the matrix $K\left(t_{i}, t_{j}\right)$. Equation (18) can be written as:

$f_{i}=\lambda \sum_{j=1}^{N} w_{j} K_{i j} f_{j}$

It is of the form $A \boldsymbol{x}=\lambda \boldsymbol{x}$. This associated set of $N$ linear algebraic equations in $N$ unknowns can be solved by standard techniques. Finding the eigenvalues and eigenvectors of the matrix $A$ is the Nystrom method. In our case, however, the kernel is singular along the diagonal line and the Nystrom method fails because of the divergence of the diagonal coefficients of the matrix $A$. One possible way out of this difficulty is to subtract the singularity in the integral. This is possible when the kernel is integrable (Press et al. 1998)

$$
\begin{aligned}
\int_{a}^{b} K_{m}(x, t) f(t) \mathrm{d} t & = \\
& \int_{a}^{b} K_{m}(x, t)[f(t)-f(x)] \mathrm{d} t+f(x) \int_{a}^{b} K_{m}(x, t) \mathrm{d} t .
\end{aligned}
$$

As a result, the singular behavior of the integrand is removed, because the logarithmic behavior in the neighborhood of the singularity implies that

$\lim _{x \rightarrow>t} K_{m}(x, t)[f(t)-f(x)]=0$.

The integral $\int_{a}^{b} K_{m}(x, t) \mathrm{d} t$ of course converges for any $x$.

Let us come back to our original problem, Eq. (15). Let $\left(\left(r_{i}\right)\right)_{i=1 . . n}$ be a subdivision of the interval $\left[r_{1}, r_{2}\right]$ with a corresponding set of weights $\left(\left(w_{i}\right)\right)_{i=1 \ldots n}$ suitable for Gauss-Legendre quadrature. Noting $\sigma_{i}=\tilde{\sigma}_{1 m}\left(r_{i}\right), \Omega_{i}=$ $\Omega_{0}\left(r_{i}\right), R_{i}=\int_{r_{1}}^{r_{2}} K_{m}\left(r_{i}, t\right) \mathrm{d} t$ and $K_{i j}=K_{m}\left(r_{i}, r_{j}\right)$, the matrix form of Eq. (15) is:

$\omega \sigma_{i}=\left(m \Omega_{i}+R_{i}-\sum_{j \neq i} w_{j} K_{i j}\right) \sigma_{i}+\sum_{j \neq i} w_{j} K_{i j} \sigma_{j}$.

Thus, our task reduces to finding the eigenvalues and eigenvectors of the matrix:

See Eq. (23) above. 
The residual function $R_{m}(r)=\int_{r_{1}}^{r_{2}} K_{m}\left(r, r^{\prime}\right) \mathrm{d} r^{\prime}$ is calculated as hypergeometric function integrals:

$$
\begin{aligned}
R_{m}(r) & =-\frac{m}{2} \frac{B_{0}}{\varepsilon_{0}} \frac{\mathrm{d}}{\mathrm{d} r}\left(\frac{\sigma_{0}}{B_{0}^{2}}\right) \frac{\Gamma\left(m+\frac{1}{2}\right)}{\Gamma(m+1) \Gamma\left(\frac{1}{2}\right)} \\
& \times\left[\int_{\frac{r_{1}}{r}}^{1} u^{m+1}{ }_{2} F_{1}\left(m+\frac{1}{2}, \frac{1}{2} ; m+1 ; u^{2}\right) \mathrm{d} r\right. \\
& +\int_{\frac{r}{r_{2}}}^{1} u^{m-2}{ }_{2} F_{1}\left(m+\frac{1}{2}, \frac{1}{2} ; m+1 ; u^{2}\right) \mathrm{d} r \\
& \left.+\frac{R_{*}^{3}}{r^{3}} \int_{\frac{R_{*}^{2}}{r_{1}}}^{\frac{R_{*}^{2}}{r_{2}}} u^{m-2}{ }_{2} F_{1}\left(m+\frac{1}{2}, \frac{1}{2} ; m+1 ; u^{2}\right) \mathrm{d} r\right]
\end{aligned}
$$

This can be done either analytically or by using a formal calculus software, like Mathematica.

\section{Results}

\subsection{Eigenspectrum}

We have computed several models of equatorial belts, with different values of the total charge $Q_{\text {tot }}$ of the system. This is normalized to the central point charge, $Q_{\mathrm{c}}=\frac{4 \pi}{3} \varepsilon_{0} \Omega_{*} B_{*} R_{*}^{3}$ (Michel 1991). Eigenvalues of a number of azimuthal modes, $m$, are shown if Fig. 4 for different charges $Q_{\text {tot }}$. The fastest growth rate is plotted versus the angular rotation velocity of the corresponding perturbation.

From this figure, it is seen that, for a given charge, the instability growth rate rises from $m=2$ to a maximum at $m \approx 18$ and then decreases slowly as $m$ increases. The instability is limited to relatively small values of $m$. It disappears for large $m$. We haven't found any instability at short wavelengths, which means that, linearly, the instability doesn't develop any fine structure. Only rather large, coherent structures emerge.

This result is confirmed by the study of the solutions of the Fredholm integral equation for large $m$. Equation (15) can be rewritten in the form:

$$
\begin{aligned}
& \left(\omega-m \Omega_{0}(r)\right) \sigma_{1 m}(r)= \\
& \quad-m F(r) \int_{r_{1}}^{r_{2}} G_{m}\left(r, r^{\prime}\right) \sigma_{1 m}\left(r^{\prime}\right) r^{\prime} \mathrm{d} r^{\prime}
\end{aligned}
$$

where $F(r)$ is a real function depending only on $\sigma_{0}$ and $B_{0}$. Figure 1 shows the shape of the Green functions $G_{m}$ for different modes $m$. It is seen that the region where $G_{m}$ is non-negligible shrinks to a very small interval around the diagonal $r=r^{\prime}$. For large $m$, let the dimension of this region be $\epsilon$. Expanding $\sigma_{1 m}\left(r^{\prime}\right)$ in a Taylor's series in the vicinity of $r$, we get:

$$
\sigma_{1 m}(r+h)=\sigma_{1 m}(r)+h \sigma_{1 m}^{\prime}(r)+\frac{h^{2}}{2} \sigma_{1 m}^{\prime \prime}(r)+o\left(h^{2}\right)
$$

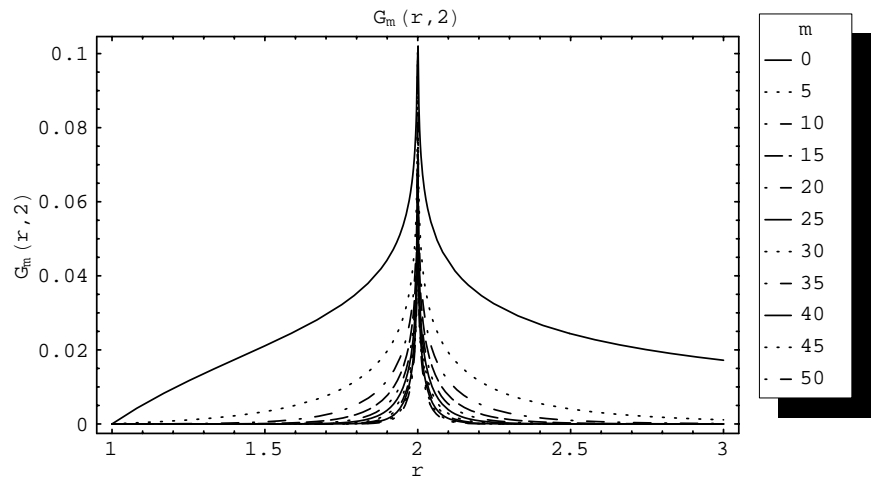

Fig. 1. The Green functions $G_{m}(r, 2)$ for different values of $m$ and for $r$ close to the source point, $r^{\prime}$, located in this case at $r^{\prime}=2$. For large values of $m, G_{m}$ decreases rapidly with $|r-2|$ and becomes negligible when $|r-2|$ becomes larger than some value, $\epsilon$.

Using this result, the integral in Eq. (24) can be approximated by:

$$
\begin{aligned}
& \int_{r_{1}}^{r_{2}} G_{m}\left(r, r^{\prime}\right) \sigma_{1 m}\left(r^{\prime}\right) r^{\prime} \mathrm{d} r^{\prime}= \\
& \sigma_{1 m}(r) \int_{r_{1}}^{r_{2}} G_{m}\left(r, r^{\prime}\right) r^{\prime} \mathrm{d} r^{\prime} \sigma_{1 m}^{\prime}(r) \int_{r_{1}}^{r_{2}} G_{m}\left(r, r^{\prime}\right) r^{\prime} h \mathrm{~d} r^{\prime} \\
& +\sigma_{1 m}^{\prime \prime}(r) \int_{r_{1}}^{r_{2}} G_{m}\left(r, r^{\prime}\right) r^{\prime} \frac{h^{2}}{2} \mathrm{~d} r^{\prime}+o\left(h^{2}\right) .
\end{aligned}
$$

Assuming $\sigma_{1 m}$ to be a smooth function with small derivatives, the first integral on the right of Eq. (26) is, in the limit of large $m$, of order $O(1)$, the second of order $O(\epsilon)$ and the third of order $O\left(\epsilon^{2}\right)$. Keeping only the dominant term, Eq. (24) becomes

$$
\omega=m \Omega_{0}(r)-m F(r) \int_{r_{1}}^{r_{2}} G_{m}\left(r, r^{\prime}\right) \sigma_{1 m}\left(r^{\prime}\right) r^{\prime} \mathrm{d} r^{\prime} .
$$

Its right hand side contains only real functions. Thus $\omega$ is real and the growth rate vanishes. The instability disappears in this limit.

On the other hand, for a fixed value of the azimuthal mode number $m$, the growth rate decreases as the charge $Q_{\text {tot }}$ of the system increases. Adding positive charge to the system has a stabilizing effect on the diocotron instability in the sense that the imaginary part of the eigenvalue progressively decreases to zero. In fact, the stabilization is not due to the value of the charge $Q_{\text {tot }}$ itself, but to the associated change in the density profile of the disk. This can be seen as follows. Suppose we replace the star by a central point charge $Q^{3}$. Its contribution to the electric drift rotation rate in the disk is

$\delta \Omega_{0}(Q)=\frac{Q}{4 \pi \varepsilon_{0} r^{3} B_{0}(r)}$.

For a dipolar magnetic field, this contribution is independent of the radius $r$ because the field strength decreases

\footnotetext{
3 Thus the boundaries conditions at the star's surface are removed and the influence of the disk's charge and his image are suppressed.
} 

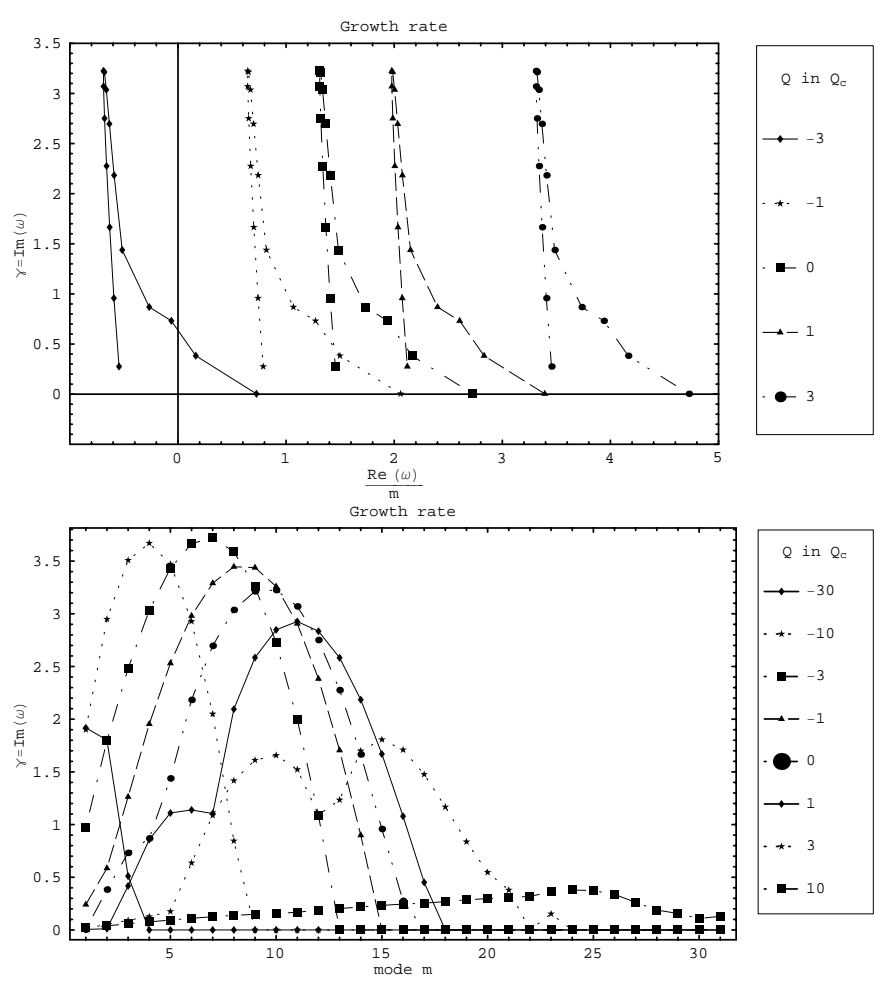

Fig. 2. The eigenvalues for a disk surrounding different central point charges $Q$, measured in units of $Q_{\mathrm{c}}$. In the figure on the top, the magnetic field is dipolar. The angular pattern speed of the perturbation is represented in abscissa and the growth rate in ordinate. In the figure on the bottom, the magnetic field threading the disk is uniform. The growth rate is represented versus the mode number $m$ for different values of $Q$.

as $1 / r^{3}$. In Eq. (15), the rotation of the disk enters only as part of the term $\left(\omega-m \Omega_{0}\right)$. Thus, if $\Omega_{0}$ is changed by a constant amount $\delta \Omega_{0}(Q)$, the corresponding eigenvalue is changed by $\delta \omega=m \delta \Omega_{0}(Q)$, which is a real number. The growth rate and the eigenfunctions are not affected by this change of rotation rate. This is illustrated in Fig. 2.

The situation changes dramatically when the dipolar magnetic field is replaced by a uniform one. The change in the rotation of the disk induced by a change of the central charge is not independent of $r$ any longer, and it can be seen that the growth rate is in this case affected. Looking at the bottom frame of Fig. 2, it can be seen that increasing the charge $Q$ to sufficiently large positive or negative values has in this case a stabilizing effect.

The influence of the geometry of the disk can be similarly investigated. We remove the central object $(Q=0)$, keeping the density profile unchanged to within a constant factor in order to maintain the total charge of the disk constant. We find that the number of unstable modes increases as the disk extension is decreased. This is summarized in Fig. 3.

This is a general trend of the diocotron instability that has already been mentioned by Davidson (1990), who investigated the stability of an electron plasma column trapped between two cylindrical conductors with an uniform axial magnetic field.

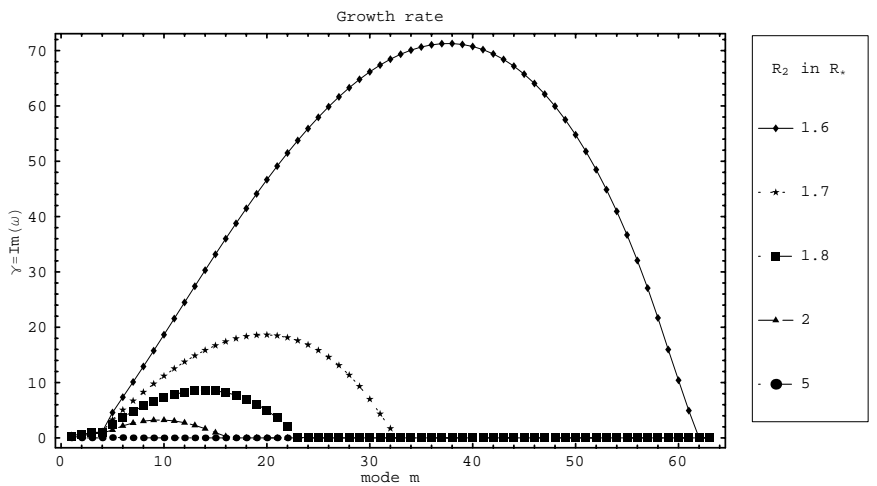

Fig. 3. The growth rates of different eigenmodes for a disk without any central charge. The disk extends from $R_{1}=1.5 R_{*}$ to different values of the outer radius $R_{2}$, as shown in the panel of the right. The density profile is the same in all case, as well as the total charge of the disk. The number of excited modes clearly decreases when $R_{2}$ increases. Beginning with $R_{2}=1.6 R_{*}$, their number are respectively $61,32,23,16$ and 18 . In the last case, the growth rate are very close to zero, less than $10^{-4}$.

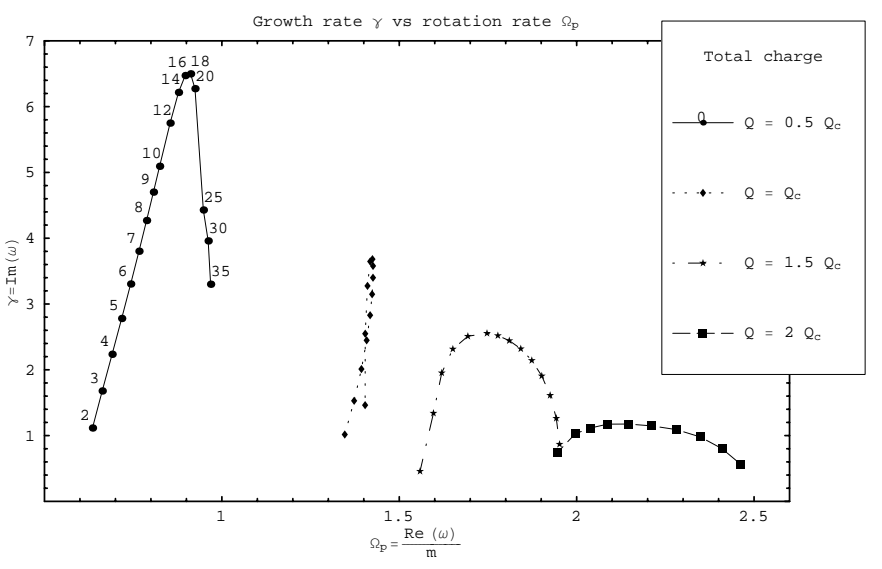

Fig. 4. Eigenvalues of the Fredholm integral Eq. (15) in the plane $\left(\gamma, \Omega_{\mathrm{p}}\right)$. For each mode $m, \gamma=\operatorname{Im}(\omega)$ is the fastest growth rate and $\Omega_{\mathrm{p}}=\operatorname{Re}(\omega) / m$ is the rotation rate of the perturbation. Each symbol corresponds to a given total charge $Q_{\text {tot }}$ as described in the insert on the right. Following the first curve, the mode $m$ increases from left to right, beginning with $m=2$ on the lower left as indicated by the numbers up to 35 at the lower right.

The growth rate of the instability is remarkably of the order of its pattern angular frequency. It develops in a very short time, much smaller than any other instabilities.

\subsection{Eigenfunctions}

Some examples of eigenfunctions are shown for two different disc models. In the first, the total charge $Q_{\text {tot }}=Q_{\mathrm{c}}$ and the disc extends in equilibrium from $r=R_{*}$ to $r=$ $1.5 R_{*}$. As can be seen in Fig. 5 , the rotation is in the range $\left[-2 \Omega_{*}, 2 \Omega_{*}\right]$ with a non-monotonic behavior, which is essential for the development of the instability. There is non negligible charge everywhere in the disc except at the extreme outer edge. The eigenfunction for $m=10$, plotted in 

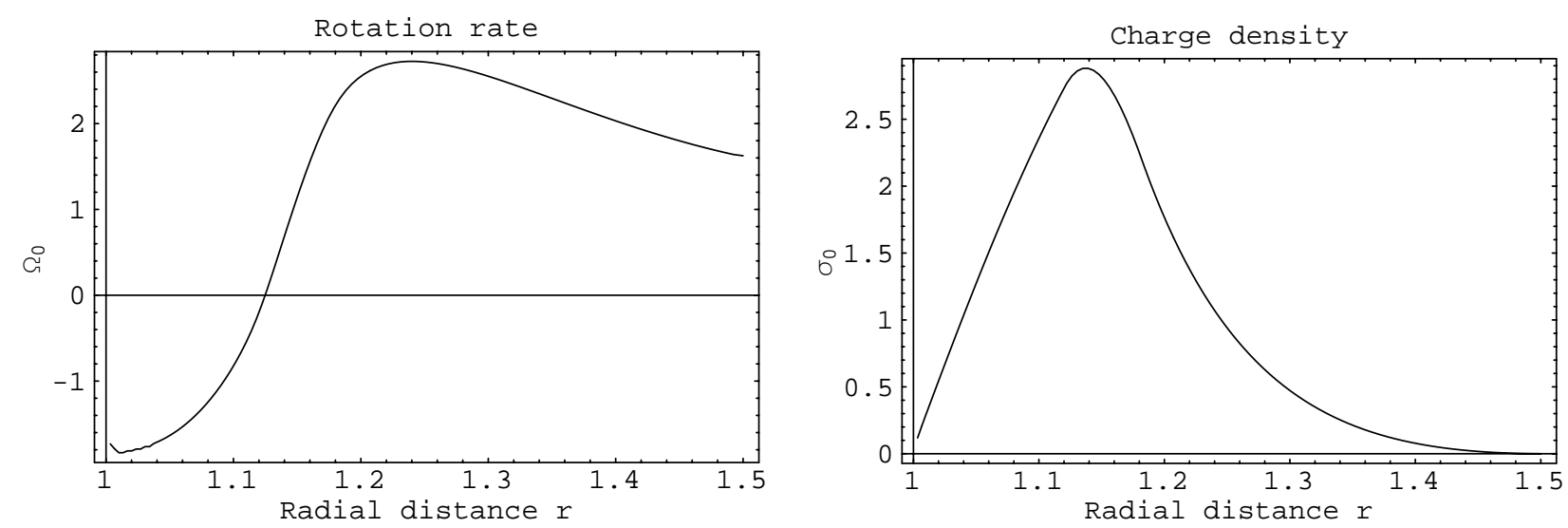

Fig. 5. Initial equilibrium state for a total charge $Q_{\text {tot }}=Q_{\mathrm{c}}$. On the left panel we show the rotation rate $\Omega_{0}(r)$ and on the right the charge density $\sigma_{0}(r)$ determined self-consistently by the system (5). The disc extends from $r=R_{*}$ to $r=1.5 R_{*}$.
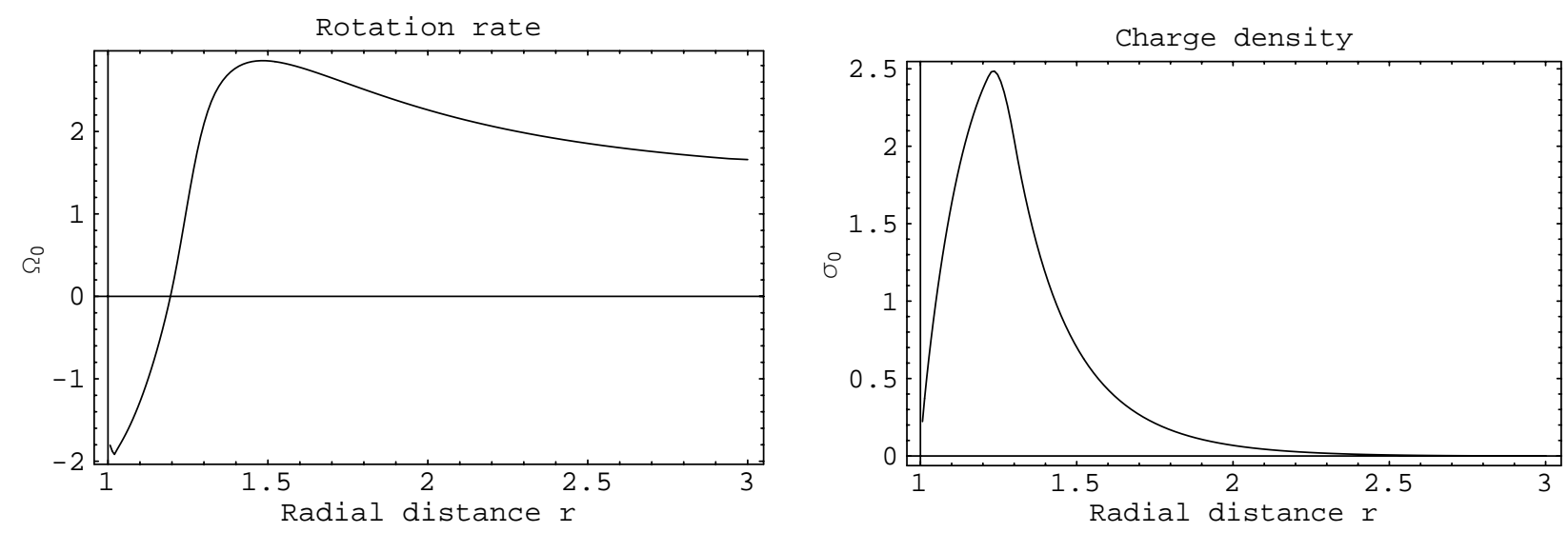

Fig. 6. Initial equilibrium state for a total charge $Q_{\text {tot }}=2 Q_{\mathrm{c}}$. On the left panel we show the rotation rate $\Omega_{0}(r)$ and on the right the charge density $\sigma_{0}(r)$. The disc extends from $r=R_{*}$ to $r=3 R_{*}$.
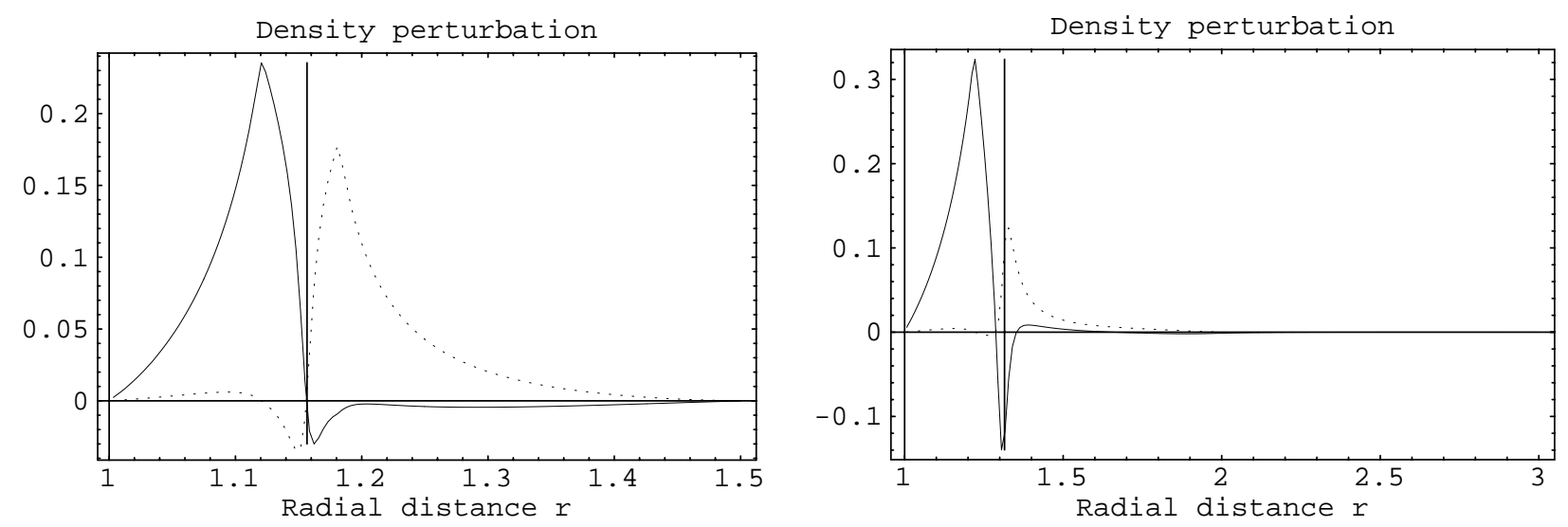

Fig. 7. Real (solid line) and imaginary (dashed line) part of eigenfunctions found from the solution of the Fredholm integral Eq. (15). On the left panel we show the fastest growing mode $m=10$ for $Q_{\text {tot }}=Q_{\mathrm{c}}$ and on the right panel we similarly show the mode $m=5$ for $Q_{\text {tot }}=2 Q_{\mathrm{c}}$. The vertical line indicates the position of corotation radius $r_{\mathrm{c}}$.

Fig. 7, vanishes exactly where the function $\frac{B_{0}}{\varepsilon_{0}} \frac{1}{r} \frac{\mathrm{d}}{\mathrm{d} r}\left(\frac{\sigma_{0}}{B_{0}^{2}}\right)$ does. Let $r_{0}$ be this particular radius. As can be seen from Eq. (12), no perturbation can develop at $r_{0}$. However the perturbation remains significant everywhere else on the disc. This is general for all eigenfunctions. The eigenfunction for $m=5$ and $Q_{\text {tot }}=2 Q_{\mathrm{c}}$ is shown in Fig. 7. The corresponding equilibrium is shown in Fig. 6. Comparing these two cases, it is seen that the perturbation is significant only in the inner half of the disc, where the charge density is large. The diocotron instability develops mainly in regions of large enough charge density, that is, in the innermost parts of the disc. This is observed for many different values of $m$ and $Q_{\text {tot }}$. The density perturbation never develops farther away from the axis than $2 R_{*}$. 

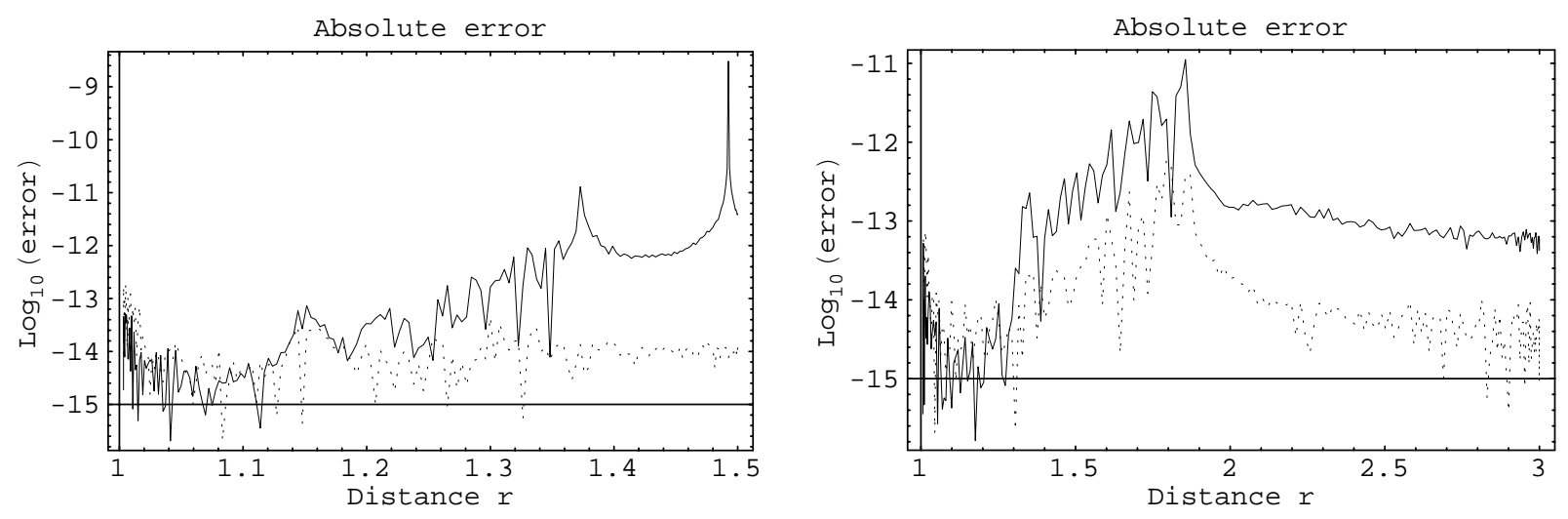

Fig. 8. Verification of the numerical algorithm used. The absolute error (as defined by (31)) between the real part of the integral transform (29) and the function (30) is shown in solid line and the corresponding error in the imaginary part is also shown in dashed line. On the left is the model $Q=Q_{\mathrm{c}}$ and $m=10$ and on the right, $Q=2 Q_{\mathrm{c}}$ and $m=5$.
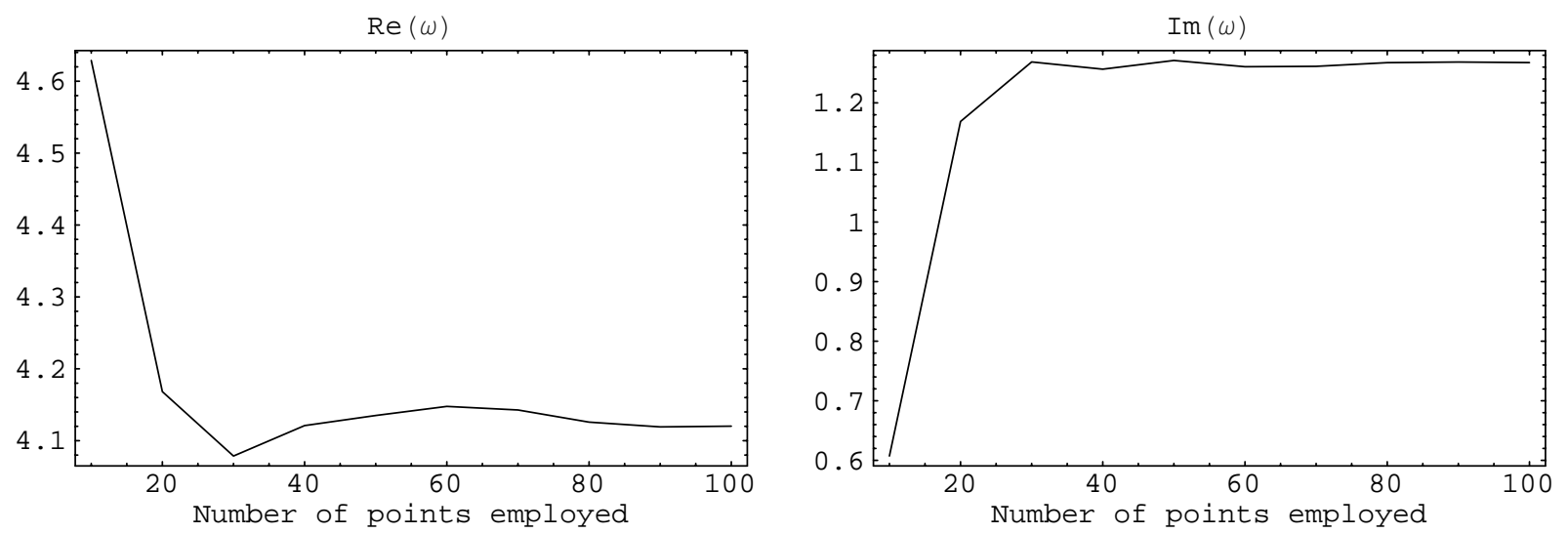

Fig. 9. Verification of the convergence to an eigenvalue. His real part on the left and his imaginary part on the right reveals the quick convergence to the value $4.12+1.26 i$ which is good approximated already by 40 points. The eigenvalue is only shown for 10 to 100 points. Beyond 100 points there is no significant change in its numerical value.

\subsection{Check of the numerical scheme}

Despite its simplicity, we felt it useful to check the reliability and efficiency of our numerical scheme. This can be done by separately computing the integral transform with the kernel $K_{m}$ on the right hand side of Eq. (15)

$\int_{r_{1}}^{r_{2}} K_{m}\left(r \mid r^{\prime}\right) \tilde{\sigma}_{1 m}\left(r^{\prime}\right) \mathrm{d} r^{\prime}$

and the function

$\left(\omega-m \Omega_{0}(r)\right) \tilde{\sigma}_{1 m}(r)$

for some peculiar eigenfunctions and eigenvalues. For example, for the eigenvector presented in Fig. 7, the absolute error, expressed as

$E_{\mathrm{abs}}=\left|\frac{\left(\omega-m \Omega_{0}(r)\right) \tilde{\sigma}_{1 m}(r)-\int_{r_{1}}^{r_{2}} K_{m}\left(r \mid r^{\prime}\right) \tilde{\sigma}_{1 m}\left(r^{\prime}\right) \mathrm{d} r^{\prime}}{\int_{r_{1}}^{r_{2}} K_{m}\left(r \mid r^{\prime}\right) \tilde{\sigma}_{1 m}\left(r^{\prime}\right) \mathrm{d} r^{\prime}}\right|$

is plotted for the real and imaginary part in Fig. 8. The accuracy of the algorithm with 200 points is better than 9 digits.

To check the convergence to an eigenvalue, we have also plotted the eigenvalues obtained numerically as a function of the number of discretization points, from 10 points to 200 points. For small $m(<20)$, the convergence is very fast, 40 or 50 points being enough to achieve results that are independent of the number of grid points. For higher $m$, like 30 or 40 , some difficulties are met due to successive numerical roundoff errors in the estimation of the residual function $R_{m}$, which involves tenths of hypergeometric functions ${ }_{2} F_{1}$. The results are in this case somewhat less accurate. An example is shown in Fig. 9.

\subsection{Two-dimensional velocity field an electric field}

As can be seen from Eq. (7), the velocity field follows the equipotentials since $\boldsymbol{v}_{1} \cdot \boldsymbol{E}_{1}=0$. Examining Figs. 10 and 12 , it seems to also follow the isodensity curves. This implies that the density perturbation $\sigma_{1}$ is proportional to the potential perturbation $\phi_{1}$. Indeed, Fig. 14, which represents the ratio between density and potential perturbation $\frac{\sigma_{1}}{\phi_{1}}$, clearly shows that this ratio is approximately constant in the two regions defined by $r \geq r_{0}$ and $r \leq r_{0}$ $\left(r_{0}=1.28\right.$ in Fig. 14). This means that the isodensities are approximately equipotentials and are thus field lines of the velocity field. Note that the plotting is inaccurate 


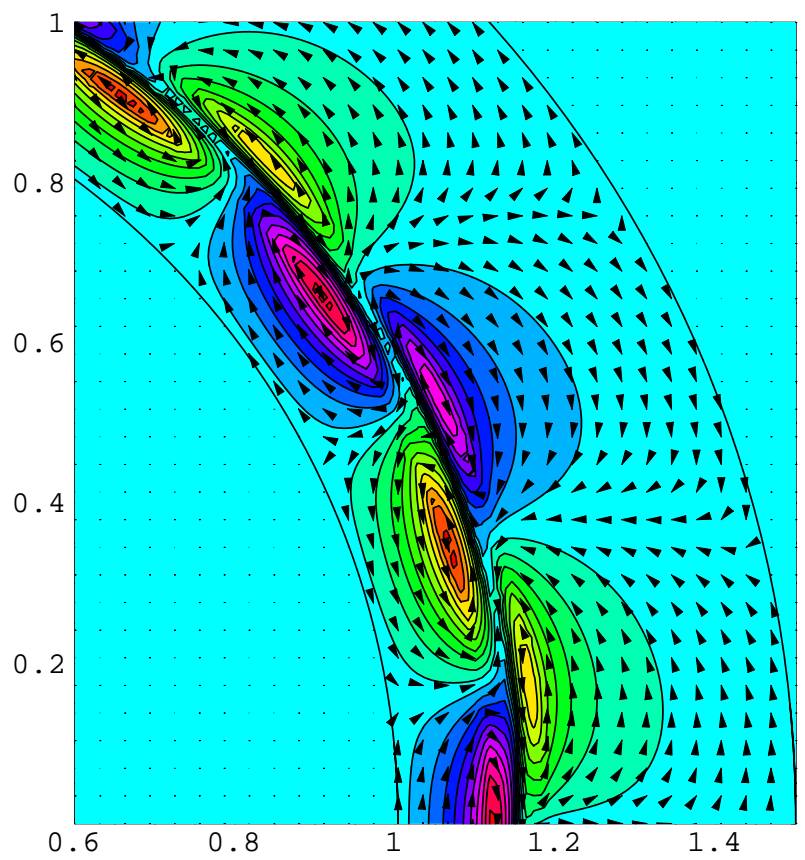

Fig. 10. Density perturbation in the disc for the mode $m=10$ and $Q=Q_{\mathrm{c}}$. The corresponding perturbed velocity field is represented by arrows. The inner and outer edges of the disc are represented by two arcs of circle of radius $R_{*}$ and $1.5 R_{*}$ respectively.

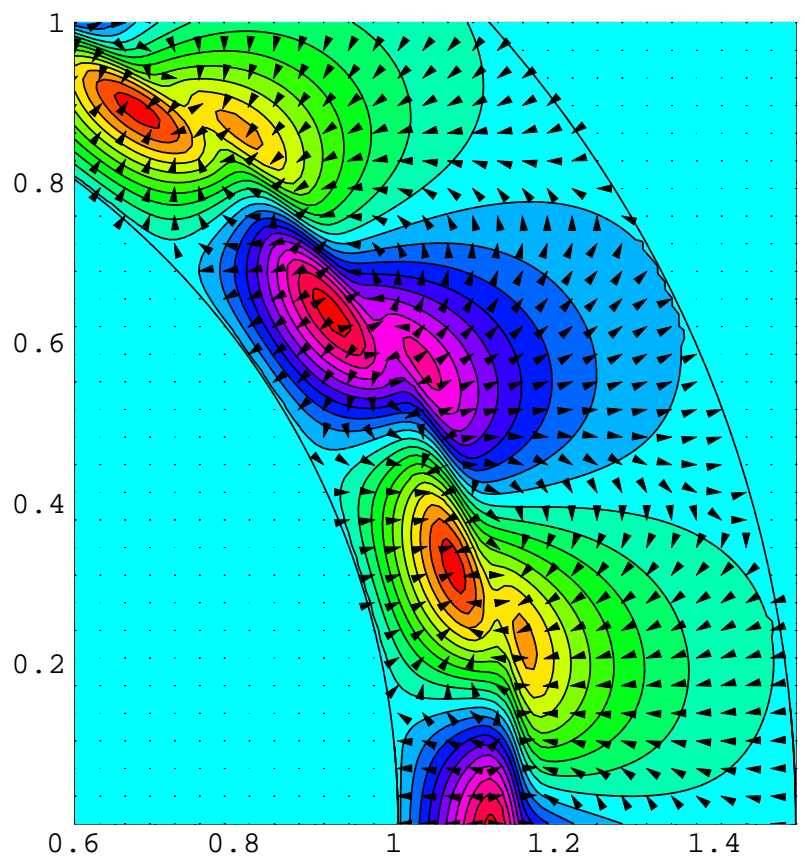

Fig. 11. Potential perturbation in the disc for the mode $m=$ 10 and $Q=Q_{\mathrm{c}}$. The corresponding perturbed electric field is represented by arrows.

when the potential approaches zero and changes sign, because the ratio $\frac{\sigma_{1}}{\phi_{1}}$ diverges there.

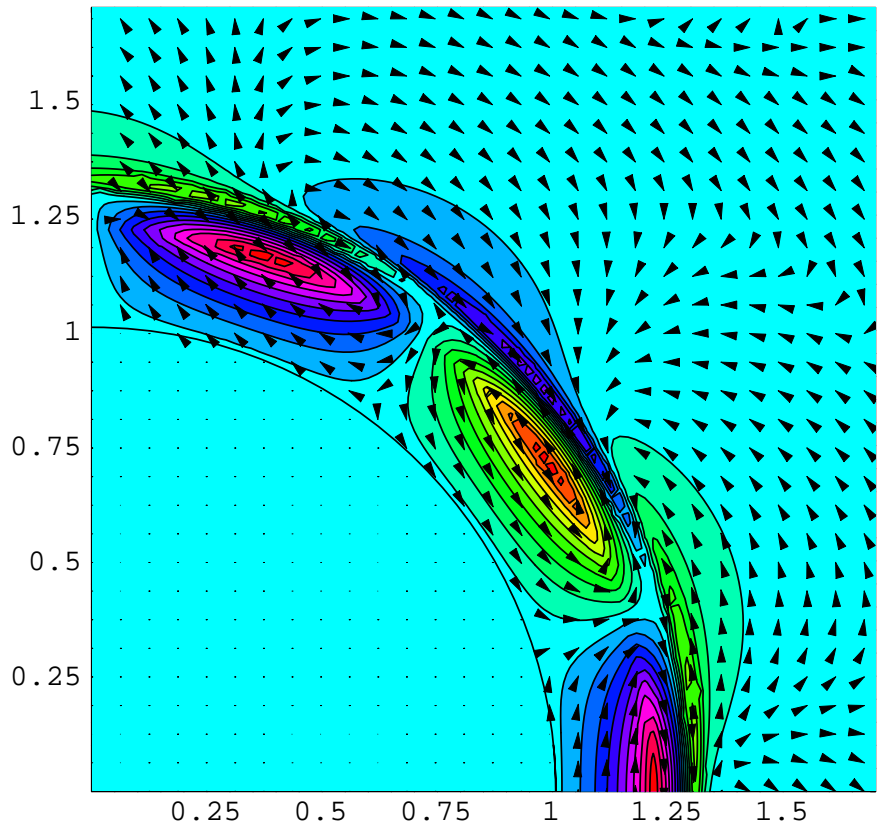

Fig. 12. Density perturbation in the disc for the mode $m=5$ and $Q=2 Q_{\mathrm{c}}$ and the corresponding perturbed velocity field. The inner edge of the disc in represented by an arc of circle of radius $R_{*}$. The outer edge, at $3 R_{*}$, is not visible on this scale.

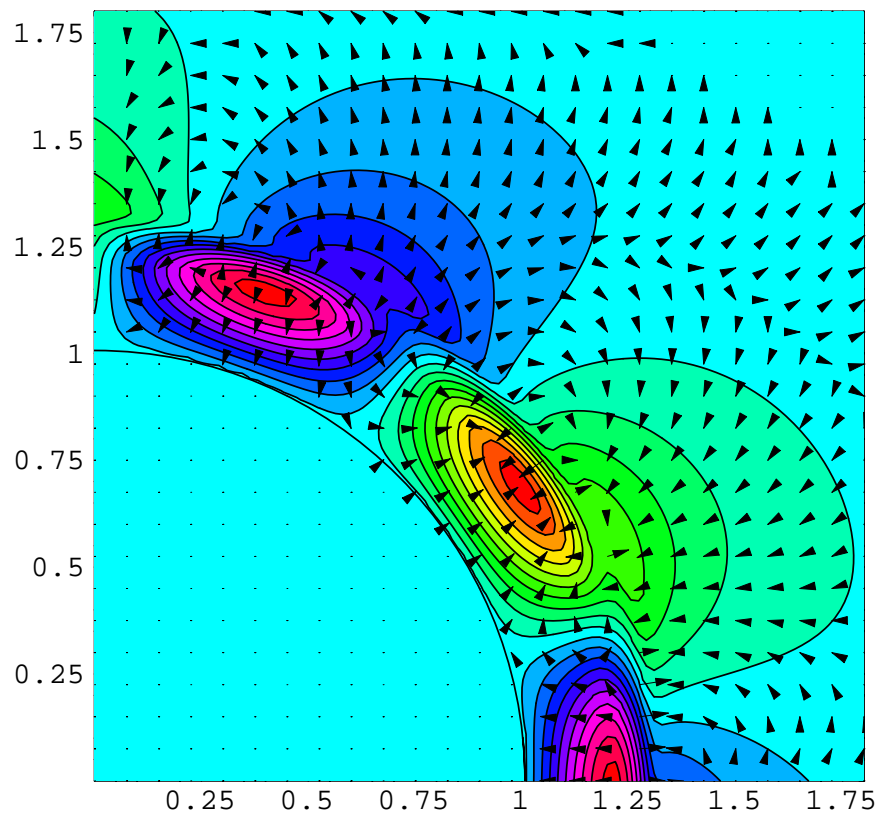

Fig. 13. Potential perturbation in the disc for the mode $m=5$ and $Q=2 Q_{\mathrm{c}}$ an the corresponding electric field. In association with the Fig. 12

\section{Conclusion}

We have shown that the equatorial charge-separated thin disc in self-consistent electrostatic equilibrium orbiting an isolated neutron star is linearly unstable to the diocotron instability. This instability develops near the inner edge 


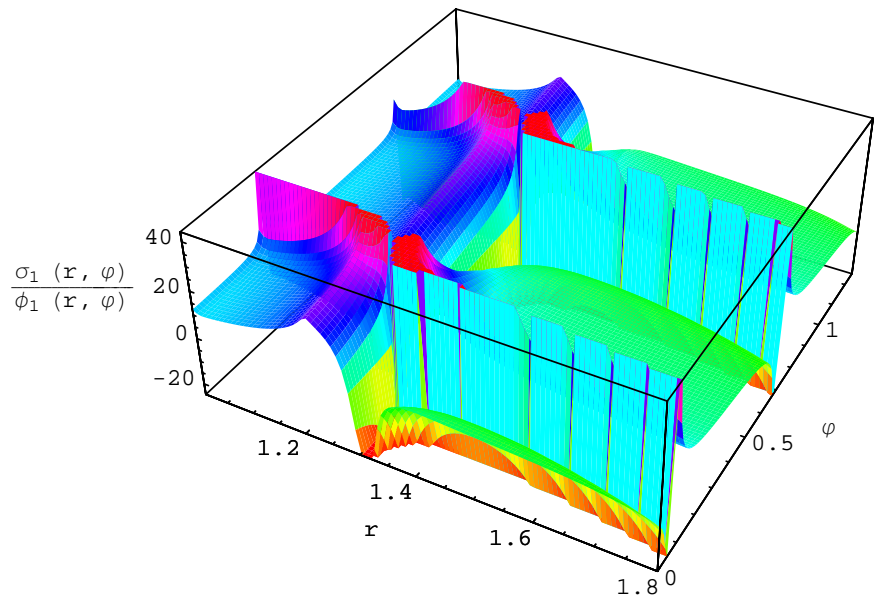

Fig. 14. The ratio of the density perturbation to the potential perturbation $\frac{\sigma_{1}(r, \varphi)}{\phi_{1}(r, \varphi)}$ for the mode $m=5$ and $Q=2 Q_{\mathrm{c}}$. Note the transition at $r_{0}=1.28$.

of the disc or, more generally, in regions of non-negligible charge density. The instability brings together particles initially located on different magnetic surfaces and it operates on very short time scales, of the order of a few neutron star rotation periods. This opens up the possibility that equatorial electric currents carry a net charge flux across field lines.

A study of the evolution of this phenomenon on long timescales requires consideration of non-linear effects. This will be done by direct numerical simulations in a forthcoming paper.

When the disc extends close to the light cylinder, the feedback of the disc electric currents on the magnetic field cannot be neglected and our electrostatic approximation fails. A fully electromagnetic instability, the magnetron instability, which also generalizes the electrostatic diocotron instability, could then develop. This will be investigated in a later paper.

Acknowledgements. We thank Christophe Pichon for help in devising a numerical method for solving the linear eigenvalue equation numerically.

\section{Appendix A: Determination of Green's function}

To make the treatment of the electrostatic problem selfconsistent, it must be demanded that the perturbed potential vanish on the star and at infinity. This is achieved by use of the well known method of images. The calculation proceeds in three steps:

- determine the potential of the disc $\Phi_{\mathrm{D}}$ which vanishes at infinity by integration of the Poisson equation;

- determine the potential of its image $\Phi_{i m \mathrm{D}}$ which vanishes at infinity;

- combine the two, so that the total potential $\left(\Phi_{\mathrm{D}}+\right.$ $\left.\Phi_{i m \mathrm{D}}\right)$ vanishes on the surface of the star.
First, we must integrate Poisson's equation in cylindrical coordinates:

$\frac{1}{r} \frac{\partial}{\partial r}\left(r \frac{\partial \Phi}{\partial r}\right)+\frac{\partial^{2} \Phi}{\partial^{2} z}-\frac{m^{2}}{r^{2}} \Phi=-\frac{\sigma}{\varepsilon_{0}} \delta(z)$

Out of the equatorial plane, this potential satisfies Laplace's equation. We look for solutions by separation of variables in the form $\Phi(r, z)=R(r) Z(z)$. Substituting this in Eq. (A.1) and dividing by $\Phi$ we get

$\frac{1}{r R} \frac{\mathrm{d}}{\mathrm{d} r}\left(r \frac{\mathrm{d} R}{\mathrm{~d} r}\right)-\frac{m^{2}}{r^{2}}=-\frac{1}{Z} \frac{\mathrm{d}^{2} Z}{\mathrm{~d} z^{2}}$.

The left hand side depends only on $r$ while the right hand side depends only on $z$. Thus the two sides must be equal to a common constant, $-k^{2}$. Then $Z(z)$ is a solution of $\frac{\mathrm{d}^{2} Z}{\mathrm{~d} z^{2}}-k^{2} Z=0$. For a potential which vanishes at $z \rightarrow \infty$, $k$ must be real. $R(r)$ is a solution of Bessel's equation:

$\frac{1}{r} \frac{\mathrm{d}}{\mathrm{d} r}\left(r \frac{\mathrm{d} R}{\mathrm{~d} r}\right)+\left(k^{2}-\frac{m^{2}}{r^{2}}\right) R=0$.

Those solutions which remain finite at the origin are the Bessel functions $J_{m}(k r)$. The particular solution for fixed $k$ and $m$ which satisfies the boundary conditions is:

$\Phi_{k m}(r)=\mathrm{e}^{-|k| z} J_{m}(k r)$.

By Coulomb's theorem, the discontinuity of its gradient at the crossing of the disc is related to the surface charge density by:

$\left.\frac{\partial \Phi_{k m}}{\partial z}\right|_{z=0^{-}}-\left.\frac{\partial \Phi_{k m}}{\partial z}\right|_{z=0^{+}}=\frac{\sigma_{k m}}{\varepsilon_{0}}$.

Taking into account the symmetry with respect to the equatorial plane we get

$\left.\frac{\partial \Phi_{k m}}{\partial z}\right|_{z=0^{+}}=-\frac{\sigma_{k m}}{2 \varepsilon_{0}}$.

By Eq. (A.4), the pair potential/density is represented in terms of Bessel functions as:

$\left\{\begin{array}{l}\sigma_{k m}(r)=2 \varepsilon_{0} k J_{m}(k r) \\ \Phi_{k m}(r)=\mathrm{e}^{-|k| z} J_{m}(k r) .\end{array}\right.$

When the surface charge density $\sigma(r, \varphi)$ is expressible as an integral transform of a set of functions $S_{m}(k)$ developed on the eigenfunctions $\sigma_{k m}$, if can be written as:

$\sigma(r, \varphi)=\sum_{m=-\infty}^{\infty} \int_{0}^{\infty} S_{m}(k) \sigma_{k m}(r) \mathrm{e}^{i m \varphi} \mathrm{d} k$.

Because Laplace's equation is linear, the potential can be similarly expanded as:

$\Phi(r, \varphi, z)=\sum_{m=-\infty}^{\infty} \int_{0}^{\infty} S_{m}(k) \Phi_{k m}(r) \mathrm{e}^{i m \varphi} \mathrm{d} k$.

By Eqs. (A.7) this gives

$\left\{\begin{array}{l}\sigma(r, \varphi)=\sum_{m=-\infty}^{\infty} \int_{0}^{\infty} 2 \varepsilon_{0} S_{m}(k) J_{m}(k r) \mathrm{e}^{i m \varphi} k \mathrm{~d} k \\ \Phi(r, \varphi, z)=\sum_{m=-\infty}^{\infty} \int_{0}^{\infty} S_{m}(k) \mathrm{e}^{-|k| z} J_{m}(k r) \mathrm{e}^{i m \varphi} \mathrm{d} k .\end{array}\right.$ 
Projecting $\sigma(r, \varphi)$ on the basis $\left(\mathrm{e}^{-i l \varphi}\right)_{l=-\infty,+\infty}$, we see that $S_{l}(k)$ is the Hankel transform of the function $\frac{1}{4 \pi \varepsilon_{0}} \int_{0}^{2 \pi} \sigma(r, \varphi) \mathrm{e}^{-i l \varphi} \mathrm{d} \varphi$. Form this we find:

$$
\begin{aligned}
S_{l}(k)= & \frac{1}{4 \pi \varepsilon_{0}} \int_{0}^{\infty} \int_{0}^{2 \pi} \sigma\left(r^{\prime}, \varphi^{\prime}\right) \mathrm{e}^{-i l \varphi^{\prime}} \\
& \times J_{l}\left(k r^{\prime}\right) r^{\prime} \mathrm{d} r^{\prime} \mathrm{d} \varphi^{\prime}
\end{aligned}
$$

Then, the potential is expressed in terms of the surfacic charge density by the following Green integral:

$$
\Phi(\boldsymbol{r})=\frac{1}{4 \pi \varepsilon_{0}} \int_{0}^{\infty} \int_{0}^{2 \pi} L\left(\boldsymbol{r} \mid \boldsymbol{r}^{\prime}\right) \sigma\left(\boldsymbol{r}^{\prime}\right) r^{\prime} \mathrm{d} r^{\prime} \mathrm{d} \varphi^{\prime} .
$$

In this spherical boundary and flat disc geometry, the Green function $L$ is:

$$
\begin{aligned}
L\left(\boldsymbol{r} \mid \boldsymbol{r}^{\prime}\right)= & \sum_{m=-\infty}^{\infty} \mathrm{e}^{i m\left(\varphi-\varphi^{\prime}\right)} \\
& \times \int_{0}^{\infty} \mathrm{e}^{-|k| z} J_{m}(k r) J_{m}\left(k r^{\prime}\right) \mathrm{d} k
\end{aligned}
$$

From this it is deduced that

$$
\begin{aligned}
\Phi_{D}(r, \varphi, z)= & \frac{1}{4 \pi \varepsilon_{0}} \int_{0}^{\infty} \int_{0}^{2 \pi} \\
& \times L\left(r, \varphi, z \mid r^{\prime}, \varphi^{\prime}\right) \sigma_{D}\left(r^{\prime}, \varphi^{\prime}\right) r^{\prime} \mathrm{d} r^{\prime} \mathrm{d} \varphi^{\prime} .
\end{aligned}
$$

The contribution from the image of the disc is simply

$$
\begin{aligned}
& \Phi_{i m D}(r, \varphi, z)=\frac{1}{4 \pi \varepsilon_{0}} \int_{0}^{\infty} \int_{0}^{2 \pi} \\
& \quad \times L\left(r, \varphi, z \mid r^{\prime}, \varphi^{\prime}\right) \sigma_{i m D}\left(r^{\prime}, \varphi^{\prime}\right) r^{\prime} \mathrm{d} r^{\prime} \mathrm{d} \varphi^{\prime} .
\end{aligned}
$$

We recall that, in order to set the potential to zero on a sphere of radius $R_{*}$, the method of images associates a point charge $q$ located at a distance $r$ from the origin with another point of charge $q^{\prime}=-\frac{R_{*}}{r} q$ located at $r^{\prime}=\frac{R_{*}^{2}}{r}$. Applying this to our disc, the charge contained in the surface element $\mathrm{d} S_{\mathrm{D}}$ of the disc, $\sigma_{\mathrm{D}}(r, \varphi) \mathrm{d} S_{\mathrm{D}}$, is imaged into a charge contained in the surface element $\mathrm{d} S_{i m \mathrm{D}}$ of the image disc by $\sigma_{i m \mathrm{D}}(r, \varphi) \mathrm{d} S_{i m \mathrm{D}}=-\frac{R_{*}}{r} \sigma_{\mathrm{D}}(r, \varphi) \mathrm{d} S_{\mathrm{D}}$.
Thus

$$
\begin{aligned}
& \Phi_{i m \mathrm{D}}(r, \varphi, z)=-\frac{1}{4 \pi \varepsilon_{0}} \int_{0}^{\infty} \int_{0}^{2 \pi} \\
& \quad \times L\left(r, \varphi, z \mid \frac{R_{*}^{2}}{r^{\prime}}, \varphi^{\prime}\right) \frac{R_{*}}{r^{\prime}} \sigma_{\mathrm{D}}\left(r^{\prime}, \varphi^{\prime}\right) r^{\prime} \mathrm{d} r^{\prime} \mathrm{d} \varphi^{\prime} .
\end{aligned}
$$

Finally, the potential of the thin disc that obeys the correct boundary conditions is ${ }^{4}$

$$
\begin{aligned}
\Phi(r, \varphi, z)= & \frac{1}{4 \pi \varepsilon_{0}} \int_{R_{*}}^{\infty} \int_{0}^{2 \pi} \\
& \times \sigma_{\mathrm{D}}\left(r^{\prime}, \varphi^{\prime}\right) G\left(r, \varphi, z \mid r^{\prime}, \varphi^{\prime}\right) r^{\prime} \mathrm{d} r^{\prime} \mathrm{d} \varphi^{\prime} .
\end{aligned}
$$

The Green function $G$ is

$$
\begin{aligned}
G\left(r, \varphi, z \mid r^{\prime}, \varphi^{\prime}\right)= & L\left(r, \varphi, z \mid r^{\prime}, \varphi^{\prime}\right) \\
& -\frac{R_{*}}{r^{\prime}} L\left(r, \varphi, z \mid \frac{R_{*}^{2}}{r^{\prime}}, \varphi^{\prime}\right) .
\end{aligned}
$$

\section{References}

Abramowitz, M., \& Stegun, I. A. 1965, Handbook of mathematical functions (Dover, N.Y.)

Arons, J. \& Smith, D. 1979, ApJ, 229, 728

Asséo, E., Pellat, R., \& Sol, H. 1983, ApJ, 266, 201

Balbus, S. A., \& Hawley, J. F. 1991, ApJ, 376, 214

Binney, J., \& Tremaine, S. 1987, Galactic dynamics (Princeton University Press)

Davidson, R. C. 1990, Physics of non neutral plasmas (Addison-Wesley Publishing Company)

Mofiz, U. A. 1992, Ap\&SS, 196, 101

Krause-Polstorff, J., \& Michel, C. F. 1985, A\&A, 144, 72

Michel, C. F. 1991, Theory of Neutron Star Magnetospheres (The University of Chicago Press)

Petri, J., Heyvaerts, J., \& Bonazzola, S. 2002, A\&A, 384, 414

Press, W., Teukolsky, S., Vetterling, W., \& Flannery, B. 1997, Numerical Recipes in C (Cambridge Univeristy Press)

Smith, I. A., Michel, F. C., \& Thacker, P. D. 2001, MNRAS, 322,209

Spitkovsky, A., \& Arons, J. 2002, to appear in Neutron stars in Supernova Remnants, ASP Conf. Proc., ed. P. O. Slane, \& B. M. Gaensler [astro-ph/0201360]

Stehle, R., \& Spruit, H. C. 2001, MNRAS, 323, 587

Weatherall, J. 1994, ApJ, 428, 261

Weatherall, J. 1997, ApJ, 483, 402

\footnotetext{
4 Another possibility would be to directly write the potential by means of the Green function of a point charge $\Phi=$ $\frac{1}{4 \pi \varepsilon_{0}} \int_{R_{*}}^{\infty} \int_{0}^{2 \pi}\left(\frac{1}{\left\|\boldsymbol{r}-\boldsymbol{r}^{\prime}\right\|}-\frac{R_{*}}{r^{\prime}} \frac{1}{\left\|\boldsymbol{r}-\frac{R_{*}^{2}}{r^{\prime}} \boldsymbol{r}^{\prime}\right\|}\right) \sigma_{\mathrm{D}}\left(r^{\prime}, \varphi^{\prime}\right) r^{\prime} \mathrm{d} r^{\prime} \mathrm{d} \varphi^{\prime}$.

This is Eq. (A.17). However expression (A.16) is more convenient because it clearly shows the dependence of the Green function on the azimuthal mode number $m$.
} 